\title{
Neuroanatomical Prediction of Anhedonia in Adolescents
}

\author{
Randy P Auerbach*,1,2, Angela Pisoni ${ }^{1,2}$, Erin Bondy ${ }^{1,2}$, Poornima Kumar ${ }^{1,2}$, Jeremy G Stewart ${ }^{1,2}$, \\ Anastasia Yendiki ${ }^{1,3}$ and Diego A Pizzagalli, 2,4
}

'Department of Psychiatry, Harvard Medical School, Boston, MA, USA; ${ }^{2}$ Center for Depression, Anxiety and Stress Research, McLean Hospital, Belmont, MA, USA; ${ }^{3}$ Athinoula A. Martinos Center for Biomedical Imaging, Department of Radiology, Massachusetts General Hospital, Boston, MA, USA; ${ }^{4}$ McLean Imaging Center, McLean Hospital, Belmont, MA, USA

\begin{abstract}
Anhedonia is a transdiagnostic risk factor implicated in mental illness onset, treatment non-response, and suicidal behaviors. Prior crosssectional research in adults has shown that anhedonia is associated with reduced dorsal striatal volume, but it is unknown whether this relationship extends to adolescents and whether reduced striatal volume prospectively predicts anhedonia. To address these gaps, the current study investigated whether striatal volume predicted anhedonia severity in adolescents. At baseline, healthy female adolescents aged I2-14 years $(n=50)$ completed a clinical assessment, and structural MRI data were acquired on a 3 Tesla MR scanner. While in the scanner, participants also completed a peer feedback task where subjective ratings following peer 'acceptance' or 'rejection' were obtained. At the three-month follow-up, participants provided self-report assessments of anhedonia, depression, and anxiety symptoms. Three main findings emerged. First, in cross-sectional analyses, right nucleus accumbens volume was inversely related to anhedonia severity. Second, reduced bilateral putamen volume prospectively predicted anhedonia severity while controlling for baseline anhedonia, depression, and anxiety symptoms. Third, a blunted subjective response to peer acceptance (ie, neutral response to positive feedback), but not a more negative subjective response to peer rejection, contributed to anhedonia severity, but only among youth with smaller putamen volume. Collectively, these results suggest that smaller volume in striatal regions critically implicated in reward processing is associated with current and future anhedonic symptoms among healthy female youth. These anatomical features may confer vulnerability to anhedonia and thus, may inform early identification of individuals at high risk for mental illness.

Neuropsychopharmacology (2017) 42, 2087-2095; doi:I0.I038/npp.20I7.28; published online 29 March 2017
\end{abstract}

\section{INTRODUCTION}

Major depressive disorder (MDD) is a leading cause of disability worldwide (Kessler, 2012) with the peak period of onset occurring during adolescence (Avenevoli et al, 2015). Approximately $74 \%$ of depressed adolescents report anhedonia (Yorbik et al, 2004), a core symptom of MDD that is insufficiently addressed by current pharmacologic (McCabe et al, 2009; McMakin et al, 2012) and psychotherapeutic (Spijker et al, 2001) interventions. For some individuals, anhedonia is a precursor to suicidal behaviors, as anhedonia severity predicts suicide completions among psychiatric adult inpatients (Fawcett et al, 1990). Among children (Nock and Kazdin, 2002) and adolescents (Auerbach et al, 2015), greater anhedonia differentiates suicide attempters from suicide ideators and thus, may be a pathway that leads to increased risk for suicidal behaviors. Given the range of negative consequences, it is essential to identify neurobiological markers that confer increased risk for anhedonia.

*Correspondence: Dr RP Auerbach, Department of Psychiatry, Harvard Medical School, Center for Depression, Anxiety and Stress Research, McLean Hospital, I I 5 Mill Street, de Marneffe, Room 240, Belmont, MA 02478, USA, Tel: +6I7 855 4405, Fax: 617855 4231,

E-mail: rauerbach@mclean.harvard.edu

Received 4 November 2016; revised 26 January 2017; accepted 27 January 2017; accepted article preview 6 February 2017

\section{Neuroanatomical Markers}

Prior research in humans has shown striatal activation modulates experiences of pleasure and reward-related behaviors (Auerbach et al, 2014; Harvey et al, 2007; Treadway and Zald, 2011). Broadly, the ventral striatum (ie, nucleus accumbens) is involved in hedonic coding and reward prediction error (Haber and Knutson, 2010), and non-human primate research has shown that unpredicted reward leads to phasic bursts of dopamine and increased learning (ie, positive prediction error), whereas reward omission leads to dopamine suppression and weakened behavior-outcome associations (ie, negative prediction error; Schultz, 2016; Schultz et al, 1997). Converging functional magnetic resonance imaging studies in humans have described robust activation in the ventral striatum in response to rewards (O'Doherty, 2004), with some research showing differential sensitivity to reward receipt (ie, increased activation; Forbes and Dahl, 2005) vs omission (ie, deactivation; O'Doherty et al, 2003; Pagnoni et al, 2002). By comparison, the dorsal striatum (ie, caudate and putamen) has been implicated in reward-related learning (Delgado, 2007), including bridging incentives to actions through stimulus-response-reward learning (O'Doherty et al, 2004). Caudate activation is strongest when the reward is unexpected-particularly when it is believed that outcomes 
are contingent on one's own actions. Finally, the putamen is a region implicated in coding of prediction errors-showing increased activation for unexpected rewards and suppression effects during omission (Haber and Knutson, 2010; Pessiglione et al, 2006).

Consistent with clinical data highlighting a prominent role of anhedonia in depressed individuals, striatal brain volume abnormalities have been described in MDD (Kempton et al, 2011; Koolschijn et al, 2009). In particular, Koolschijn and colleagues' meta-analysis showed small to moderate volume reductions in the caudate (Cohen's $d=-0.31$ ) and putamen (Cohen's $d=-0.48$ ) among depressed patients relative to healthy individuals. More recently, Kempton and colleagues confirmed these findings and also found reduced volume within the caudate (Hedges' $g=-0.25$ ) and putamen (Hedges' $g=-0.30$ ) in depressed relative to bipolar disorder patients. Less research, however, has tested neuroanatomical markers of anhedonia. Nonetheless, cross-sectional studies have shown an inverse relationship between trait anhedonia and caudate volume in healthy adults (Harvey et al, 2007) and unmedicated depressed adults (Pizzagalli et al, 2009). Despite these initial insights, it is unknown whether: (1) this relationship is present early in life, including before rates of MDD begin to surge (Avenevoli et al, 2015) and (2) striatal brain volume prospectively predicts anhedonia.

\section{Peer Feedback and Brain Volume Abnormalities}

Adolescence is characterized by greater autonomy from parents and enhanced reliance on peers, which affords increased opportunities for peer acceptance and rejection (Guyer et al, 2012). Indeed, adolescents experience a greater frequency of interpersonal stress-particularly as it relates to peer rejection-relative to children and adults (Rudolph, 2008), and such stress is a strong predictor of anhedonia (Auerbach et al, 2014; Pizzagalli, 2014). Research across animals and humans has provided evidence that social stress negatively affects reward processes. For example, chronically stressed animals show reduced appetitive behaviors and social motivation (eg, Katz et al, 1981; Lucas et al, 2004). Along these lines, healthy adults with a history of child maltreatment were characterized by lower subjective ratings of reward cues as well as reduced striatal activation following reward (but not penalty) anticipation (Dillon et al, 2009; Mehta et al, 2010). Although neuroimaging research has shown that peer acceptance and rejection elicit distinct patterns of striatal activation (Guyer et al, 2009, 2012), considerably less is known about whether striatal volume impacts response to peer feedback.

\section{Goals of the Current Study}

The goal of the current study was to test the prospective relationship between striatal volume and anhedonia among adolescents. First, in line with prior research (Harvey et al, 2007; Pizzagalli et al, 2009), we hypothesized that reduced dorsal (caudate and putamen) but not ventral (nucleus accumbens) striatum volume would prospectively predict anhedonia severity while controlling for baseline anhedonia, depression, and anxiety symptoms. Second, given the importance of peer relationships in adolescent development, exploratory analyses tested whether striatal brain volume would moderate the relationship between subjective response to peer feedback and greater anhedonia severity.

\section{MATERIALS AND METHODS}

\section{Participants}

The study included 50 healthy, female adolescents aged 1214 years $(M=13.04, \mathrm{SD}=0.83)$. To minimize neuroanatomical and pubertal heterogeneity only female participants were included. Further, female youth experience twice as many depressive episodes relative to male adolescents, and the selected age range precedes the peak onset of MDD (Avenevoli et al, 2015). A baseline assessment before the surge depression was deemed important to prospectively predict anhedonia without the potential confound of current psychiatric symptoms. Tanner stage pubertal variation was minimal $(M=3.28, \mathrm{SD}=0.49)$, and participants endorsed the following ethnicities: 78\% White, 6\% Asian, 4\% Black, and $12 \%$ more than one race. The distribution of family incomes included: $62 \%$ greater than $\$ 100,000,14 \% \$ 75,000-\$ 100,000$, $8 \% \$ 50,000-\$ 75,000,2 \% \$ 25,000-50,000$ (14\% did not report their family income).

At the 3-month follow-up, no differences emerged when comparing the follow-up completers $(n=37)$ and attriters $(n=13)$ in age $(t(48)=1.79, p=0.08, d=0.52)$, Tanner stage $(t(48)=-0.44, p=0.66, d=-0.13)$, race $\left(\chi^{2}(3, n=50)=6.55\right.$, $p=0.09, \varphi=0.36)$, or family income $\left(\chi^{2}(4, n=50)=5.04\right.$, $p=0.28, \varphi=0.32)$. In addition, there were no differences among follow-up completers and attriters in baseline anhedonia $(t(48)=-1.07, p=0.29, d=-0.31)$, depression $(t(48)=-0.61, p=0.55, d=-0.18)$, or anxiety $(t(48)=$ $-1.24, p=0.22, d=-0.36)$ symptoms.

\section{Procedure}

The Partners Institutional Review Board provided approval for the study. Adolescents assented, and a legal guardian provided signed consent. Inclusion criteria included English fluency, female sex, and right-handedness, and exclusion criteria were history of psychiatric illness, psychotropic medication use, neurological disorders, or seizures. The initial procedures were completed over two laboratory visits. On the first visit, adolescents were administered a diagnostic interview, self-report measures, and the first part of the Chatroom Task. During the second visit, which occurred within $1-2$ weeks (median $=8$ days, $\mathrm{SD}=6.41$ ), structural MRI data were acquired on a Siemens Tim Trio 3.0 Tesla scanner and behavioral data from the Chatroom Task feedback phase also were obtained (while in the scanner). At the 3-month follow-up, participants completed self-report measures of psychiatric symptoms. As this is a developmental stage characterized by increased social stress, prior research has demonstrated that a 3-month period is sufficient to probe fluctuations in associated mood and anxiety symptoms (Auerbach and Ho, 2012). Participants were remunerated $\$ 100$.

\section{Clinical Instruments}

Diagnostic interview. The Schedule for Affective Disorders and Schizophrenia for School-Age Children-Present 
(K-SADS-PL; Kaufman et al, 1997) assessed DSM-IV disorders. All interviews were recorded, and the principal investigator (RPA) selected $20 \%$ of the interviews to assess inter-rater reliability. The Cohen's kappa coefficients were excellent $(\kappa=1.00)$.

Pubertal status. The Tanner Scale (Tanner and Davies, $1985)$ is a self-report assessment of physical development. The 5-item instrument assesses external primary and secondary sex characteristics. The current study used the female pubertal status instrument.

Anhedonia symptoms. The Snaith-Hamilton Pleasure Scale (SHAPS; Snaith et al, 1995) is a 14-item self-report inventory. Item scores range from 1 (strongly disagree) to 4 (strongly agree). Items were reverse coded, and total scores can range from 14 to 56 with higher scores indicating greater symptom severity. Previous research with adolescents has demonstrated strong psychometric properties (Auerbach et al, 2015). The internal consistency was excellent (baseline $\alpha=0.88$; follow-up $\alpha=0.89$ ).

\section{Depression symptoms}

The Mood And Feelings Questionnaire. Long Version (MFQ; Angold et al, 1995) is a 33-item self-report measure assessing depressive symptoms in the past 2 weeks. For each item, participants rated whether a statement was 0 (not true), 1 (sometimes true), or 2 (true). Total scores range from 0 to 66 , and higher scores reflect more severe depression severity. Internal consistency was excellent (baseline $\alpha=0.93$; followup $\alpha=0.88$ ).

Anxiety symptoms. The Multidimensional Anxiety Scale for Children (MASC; March et al, 1997) is a 39-item selfreport inventory. Item scores range from 0 (never true about me) to 3 (often true about me) with total scores ranging from 0 to 117 , and higher scores indicate greater anxiety severity. Internal consistency was excellent (baseline $\alpha=0.81$; followup $\alpha=0.89$ ).

\section{Experimental Task}

The Chatroom Task (Guyer et al, 2009, 2012) was designed to simulate adolescent social interactions and to probe differential response to peer feedback (ie, acceptance $v s$ rejection). E-Prime (Psychological Software Tools, Pittsburgh, PA) software was used to present stimuli and record responses. In phase 1 , participants were led to believe they were participating in a nationwide study of how adolescents interact in online chatrooms. First, they created an online profile (ie, indicating likes and dislikes) and then, accompanying photographs of the participants were taken. Next, they viewed photographs of 60 same-aged female adolescents and selected 30 adolescents they were 'interested' and 'not interested' in chatting with online following a neuroimaging scan 1-2 weeks later. Participants were informed that female peers from collaborating institutions would review their profiles and indicate whether they were interested (ie, peer acceptance) or not interested (ie, peer rejection) in chatting online. Participants viewed pictures but not profiles of other adolescents.
For phase 2, participants received peer feedback from the 60 female adolescents allegedly participating in the nationwide study while fMRI data were acquired (The Chatroom Task was completed while functional neuroimaging data were collected. These fMRI data will be reported in a separate line of work probing social reward processes in adolescents.). During each trial, a participant viewed the photograph of a 'participating female adolescent' $(1300 \mathrm{~ms})$, and a photograph caption displaying interested or not interested was used to remind a participant about their prior selection. Then, a jittered fixation cross (1300-7600 ms) was presented, which was followed by the peer feedback superimposed under the photograph $(2600 \mathrm{~ms})$. After the feedback, a jittered fixation cross (1300-5200 ms) was displayed, and next a participant received a prompt, 'How does this make you feel?,' and was instructed to provide a rating on a visual analogue scale ranging from 0 (very bad) to 100 (very good). Feedback was provided in pseudorandom order with no more than 3 trials of the same response provided consecutively. Unbeknownst to the participants, feedback was fixed, as all participants received the same number of acceptance (30 interested trials) and rejection (30 not interested trials) trials. For the current study, the average subjective rating for peer acceptance (interested; $M=64.87$, $\mathrm{SD}=9.88$ ) and rejection (not interested; $M=46.14, \mathrm{SD}=$ 9.44) were used to test study hypotheses.

After completing the Chatroom Task, participants were debriefed. To determine whether the task manipulation was believable, the experimenter asked a series of questions that participants rated on a scale from 1 to 10 (higher scores reflect more positive responses): (1) 'How interested were you in this task?' ( $M=6.67$; $\mathrm{SD}=1.98)$; (2) 'How happy were you when someone expressed interest in chatting with you?' $(M=7.80, \mathrm{SD}=1.69)$; (3) 'How angry were you when someone rejected you?' $(M=3.20, \mathrm{SD}=1.99)$; and (4) 'How nervous did you feel while waiting for the other person to make their choice?' $(M=4.01, \mathrm{SD}=2.37)$. Outlier criterion was defined as $\pm 3 \mathrm{SD}$ on any item response; no participant data were removed based on these criteria.

\section{Image Acquisition}

Structural MRI data were acquired on a Siemens Tim Trio 3.0 Tesla MR scanner equipped with a 32-channel head coil. High-resolution structural data were collected using a multiecho magnetization-prepared gradient echo (MEMPRAGE) sequence with the following parameters: $1.2 \mathrm{~mm}$ isotropic resolution; TR $2200 \mathrm{~ms}$; TE1 $1.54 \mathrm{~ms}$; TE2 $3.36 \mathrm{~ms}$; TE3 $5.18 \mathrm{~ms}$; TE4 $7.01 \mathrm{~ms}$; TI $1100 \mathrm{~ms}$; flip angle $7^{\circ}$; BW $651 \mathrm{~Hz} /$ Px; GRAPPA factor 4; matrix size $192 \times 192 \times 144$.

\section{Data Processing and Analytic Overview}

The structural images were processed with Freesurfer (http:// surfer.nmr.mgh.harvard.edu) to obtain an automated cortical parcellation and subcortical segmentation (Fischl et al, 2004). Data analysis focused on volume $\left(\mathrm{mm}^{3}\right)$ in striatal regions (putamen, caudate, and nucleus accumbens) (Figure 1a).

All statistical analyses were conducted using IBM SPSS Statistics Version 20. Dependent variables were tested for skewness $( \pm 1.5)$. Only baseline and 3-month follow-up depression scores were skewed and thus, normalized using 
a

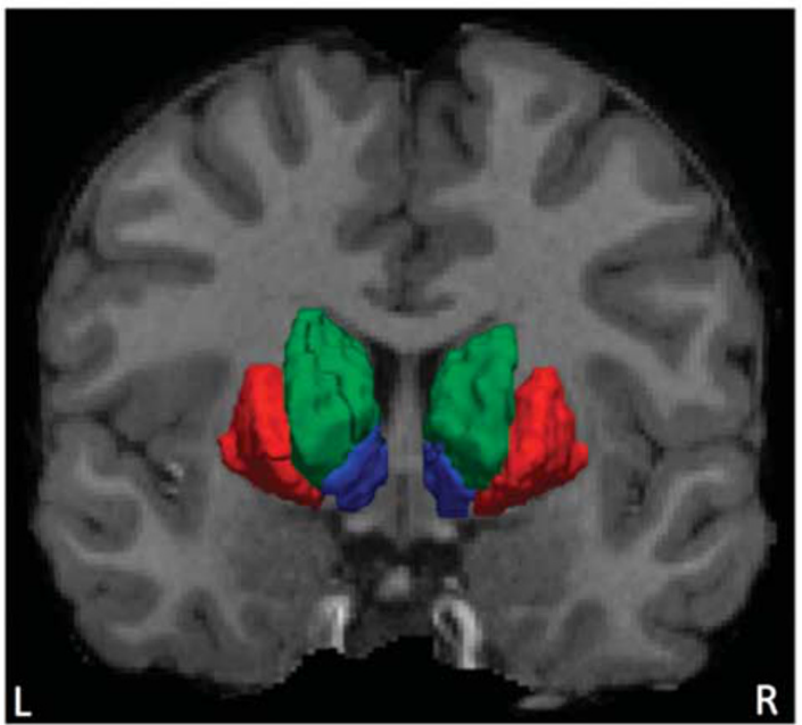

b

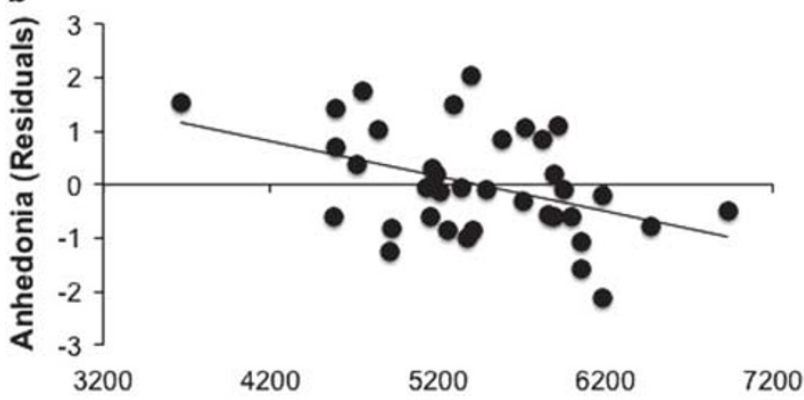

Left Putamen Volume

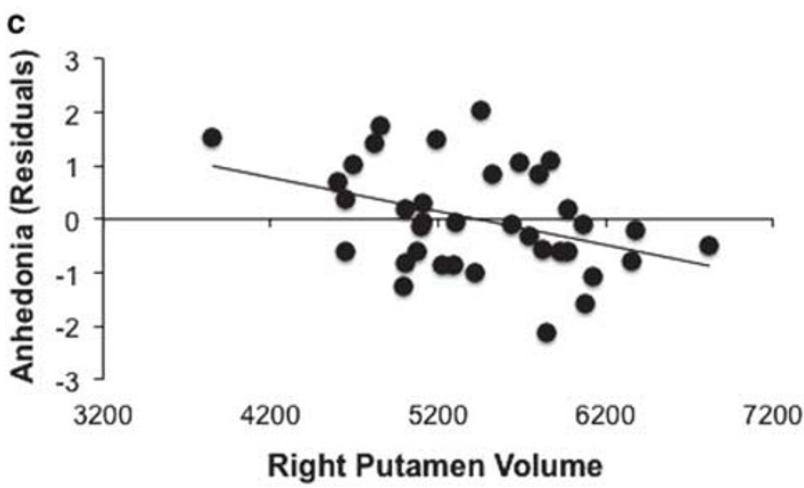

Figure I Bilateral putamen volume prospectively predicts anhedonia at the 3-month follow-up assessment $(n=37)$. (a) Striatal regions of interest, include: (i) putamen (red), (ii) caudate (green), and (iii) nucleus accumbens (blue); left (b) and (c) right putamen volume $\left(\mathrm{mm}^{3}\right)$ predicts anhedonic symptoms (SHAPS score) at the 3-month follow-up assessment (standardized residuals).

a square root transformation. Primary analyses focused on anhedonia as an outcome variable. Secondary analyses tested the specificity of putative findings and explored depression and anxiety symptoms as dependent variables. For all analyses, we ran a series of stepwise linear regression models. In step 1, total intracranial volume was included as a covariate. In cross-sectional models, all symptoms other
Table IA Pearson Correlations, Means, And SD's Of Striatal Volume $\left(\mathrm{mm}^{3}\right)$ Among Female Adolescents $(n=50)$

\begin{tabular}{lrrrrrr}
\hline & $\mathbf{I}$ & $\mathbf{2}$ & $\mathbf{3}$ & $\mathbf{4}$ & $\mathbf{5}$ & $\mathbf{6}$ \\
\hline I. Left putamen & - & & & & & \\
2. Right putamen & $0.98^{* * *}$ & - & & & & \\
3. Left caudate & $0.7 I^{* * *}$ & $0.71^{* * *}$ & - & & & \\
4. Right caudate & $0.67^{* * *}$ & $0.66^{* * *}$ & $0.96^{* * *}$ & - & & \\
5. Left nucleus & $0.29 *$ & $0.33^{*}$ & 0.25 & $0.30^{* *}$ & - & \\
accumbens & & & & & & \\
6. Right nucleus & $0.68^{* * *}$ & $0.70^{* * *}$ & 0.61 & $0.55^{* * *}$ & $0.60^{* * *}$ & - \\
accumbens & & & & & & \\
& & & & & & \\
Mean (mm ${ }^{3}$ ) & 5439.22 & 5424.34 & 4049.11 & 4137.92 & 373.53 & 663.09 \\
SD & 617.32 & 604.33 & 501.04 & 489.68 & 94.31 & 115.90 \\
Min & 3660.70 & 3850.70 & 3066.50 & 2987.00 & 224.30 & 463.40 \\
Max & 6930.60 & 6820.40 & 5388.10 & 5582.10 & 608.10 & 920.40 \\
Range & 3269.90 & 2969.70 & 2321.60 & 2595.10 & 383.80 & 457.00 \\
\hline
\end{tabular}

* $p<0.05$, ***** $p<0.001$.

Table IB Pearson Correlations, Means, And SD's Of Psychiatric Symptoms At The Initial $(n=50)$ And Follow-up $(n=37)$ Assessment

\begin{tabular}{lrrrrrr}
\hline & I & $\mathbf{2}$ & $\mathbf{3}$ & $\mathbf{4}$ & $\mathbf{5}$ & $\mathbf{6}$ \\
\hline I. Initial anhedonia & \multicolumn{1}{c}{-} & & & & & \\
2. Initial depression & 0.25 & - & & & & \\
3. Initial anxiety & 0.16 & $0.38^{* *}$ & - & & & \\
4. Follow-up anhedonia & $0.56^{* * *}$ & 0.19 & 0.0 & - & & \\
5. Follow-up depression & $0.45^{* * *}$ & $0.83^{* * *}$ & $0.44^{* *}$ & 0.15 & - & \\
6. Follow-up anxiety & $0.38^{*}$ & 0.23 & $0.66^{* * *}$ & 0.04 & $0.52^{* * *}$ & - \\
& & & & & & \\
Mean & 20.02 & 6.87 & 38.16 & 19.38 & 4.88 & 34.40 \\
SD & 4.59 & 7.86 & 11.32 & 4.44 & 5.98 & 13.68 \\
Min & 14.00 & 0.00 & 14.00 & 14.00 & 0.00 & 11.00 \\
Max & 34.00 & 42.00 & 73.00 & 27.00 & 23.00 & 76.00 \\
Range & 20.00 & 42.00 & 59.00 & 13.00 & 23.00 & 65.00 \\
\hline
\end{tabular}

* $p<0.05$, *** $p<0.01$, **** $p<0.00$ I. Note: Mood and Feelings Questionnaire $=$ depression; Multidimensional Anxiety Scale for Children $=$ anxiety; Snaith-Hamilton Pleasure Scale $=$ anhedonia.

than the outcome symptom measure also were entered as covariates. In prospective analyses, however, all baseline symptoms were included as covariates. Pubertal status was not included as a covariate, as it was not associated with any symptom outcome at the baseline ( $r s=-0.06-0.24$, $p s>0.13)$ or the follow-up ( $r s=-0.01-0.13, p s>0.46$ ) assessment. In Step 2, we entered the independent variable, which included the putamen, caudate, or nucleus accumbens volume. Given high correlations among striatal subregions (see Table 1A and B), effects for each region of interest were tested separately. The dependent variable was baseline or 3-month follow-up anhedonia, depression, or anxiety 
a
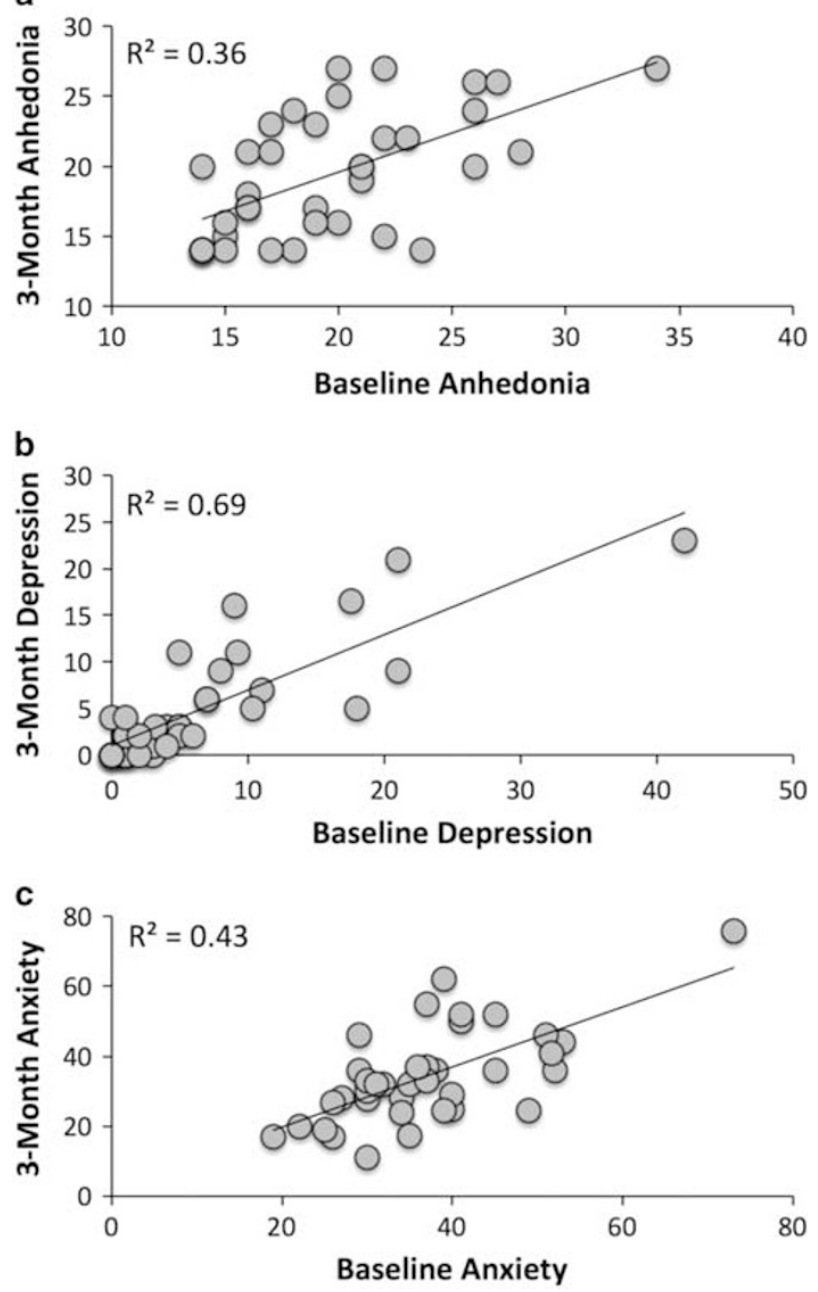

Figure 2 Scatterplots of baseline and 3-month follow-up anhedonia, depression, and anxiety symptoms. Scatterplots include baseline and 3-month follow-up assessment for the: (a) Snaith-Hamilton Pleasure Scale = anhedonia; (b) Mood and Feelings Questionnaire=depression; (c) Multidimensional Anxiety Scale for Children = anxiety.

symptoms, which were entered in separate models to test the specificity of findings.

We also estimated series of moderation models using Model 1 from version 2.15 of PROCESS (Hayes, 2013) by testing whether striatal volume moderated the relationship between the subjective response to peer feedback and symptom severity at the 3-month follow-up. We included total intracranial volume and all baseline symptoms as covariates. The independent variable was the average subjective response to peer feedback, and moderators, which were entered separately, included the putamen, caudate, or nucleus accumbens volume. All models included 5000 bootstrap samples with a bias corrected bootstrap confidence interval. Products in the model were mean centered, and heteroscedasticity-consistent standard errors were estimated. A Johnson-Neyman's region of significance approach probed significant interactions to determine the point at which subcortical volume strengthens the association between peer feedback and symptom severity.

\section{RESULTS}

Pearson correlations, means, and SD's for baseline and follow-up measures are summarized in Table $1 \mathrm{~A}$ and $\mathrm{B}$, and scatterplots of baseline and follow-up symptoms are provided in Figure 2. There were no significant differences between baseline and follow-up assessments for anhedonia, $t(36)=0.35, p=0.73, d=0.16$, or anxiety, $t(36)=1.51$, $p=0.14, d=0.36$, symptoms. There was a modest reduction in depression symptoms over time, $t(36)=2.04, p=0.049$, $d=0.50$, although scores were well below the range of clinical significance.

\section{Primary Analyses: Subcortical Volume and Anhedonia}

Cross-sectional analyses. To predict baseline anhedonia, depression, anxiety, and total intracranial volume were included as covariates. The putamen $(p s>0.15)$, caudate $(p s>0.51)$, and left nucleus accumbens $(p=0.92)$ volume were not associated with anhedonia symptoms. However, there was a significant relationship between smaller right nucleus accumbens volume and greater anhedonia severity $(b=-0.45, p=0.01, p r=-0.37)$.

Prospective analyses. When predicting 3-month anhedonic symptoms, baseline symptoms and total intracranial volume were included as covariates. Smaller left and right putamen volume predicted greater anhedonia severity, accounting for 53 and $51 \%$ of the variance in the outcome variable, respectively (Table 2, Figure $1 \mathrm{~b}$ and $c$ ). Neither caudate $(p s>0.35)$ or nucleus accumbens ( $p s>0.63)$ volume predicted anhedonic symptoms.

Moderation analyses. Analyses tested whether volume of subcortical structures moderated the relationship between peer feedback and anhedonia severity at the 3month follow-up. The inclusion of the putamen $\times$ acceptance interaction improved the model (left putamen: $\Delta \mathrm{F}(1,29)=4.90, \quad p=0.03, \quad \Delta R^{2}=0.06$; right putamen: $\left.\Delta \mathrm{F}(1,29)=5.16, p=0.03, \Delta R^{2}=0.05\right)$ (Table 3). A JohnsonNeyman's region of significance approach was then utilized to probe this interaction. When the left putamen volume is $4882.11 \mathrm{~mm}^{3}$, the relationship between blunted peer acceptance (ie, a more neutral rating in response to positive feedback) and anhedonia severity are significantly related, $\quad b=-0.20, \quad \mathrm{SE}=0.10, \quad t=-2.05, \quad p=0.05$, $\mathrm{CI}_{95}=-0.40,0.00$. As the putamen volume decreases to $3663.17 \mathrm{~mm}^{3}$, the relationship between peer acceptance and anhedonic symptoms becomes markedly stronger, $b=-0.51$, $\mathrm{SE}=0.22, t=-2.27, p=0.03, \mathrm{CI}_{95}=-0.97,-0.05$. Similarly, for the right putamen, the interaction becomes significant at $4959.19 \mathrm{~mm}^{3}, \quad b=-0.16, \quad \mathrm{SE}=0.08, \quad t=-2.05, \quad p=0.05$, $\mathrm{CI}_{95}=-0.33,0.00$, and the association between blunted peer acceptance and anhedonia severity is most pronounced when the putamen volume is $3843.10 \mathrm{~mm}^{3}, b=-0.42, \mathrm{SE}=0.17$, $t=-2.39, p=0.02, \mathrm{CI}_{95}=-0.78,-0.06$.

Highlighting the specificity of these effects: (a) the putamen $\times$ acceptance interaction remained significant while also controlling for peer rejection in the left putamen $(p=0.048)$ and trended in the expected direction in the right putamen $(p=0.054)$ and (b) no significant interaction emerged when testing the putamen $\times$ rejection interaction 
Table 2 Bilateral Putamen Volume Predicts Anhedonia Severity $(n=37)$

\begin{tabular}{|c|c|c|c|c|c|}
\hline & $b$ (SE) & $\boldsymbol{t}$ & $p$ & $\mathrm{Cl}_{95}$ & pr \\
\hline \multicolumn{6}{|c|}{ Left/right putamen step I: $\Delta F(4,32)=5.16, p=0.003 ; \Delta R^{2}=0.39$} \\
\hline Baseline anhedonia & $0.58(0.14)$ & 4.08 & $<0.001$ & $0.29,0.86$ & 0.59 \\
\hline Baseline depression & $-0.01(0.08)$ & -0.11 & 0.91 & $-0.18,0.16$ & -0.02 \\
\hline Baseline anxiety & $-0.05(0.06)$ & -0.78 & 0.44 & $-0.17,0.08$ & -0.14 \\
\hline Total intracranial volume ${ }^{\mathrm{a}}$ & $-0.83(0.70)$ & -1.19 & 0.24 & $-2.25,0.59$ & -0.21 \\
\hline \multicolumn{6}{|c|}{ Left putamen step 2: $\Delta \mathrm{F}(1,31)=8.82, p=0.006 ; \Delta \mathrm{R}^{2}=0.14$} \\
\hline Baseline anhedonia & $0.44(0.13)$ & 3.31 & 0.002 & $0.17,0.72$ & 0.51 \\
\hline Baseline depression & $-0.01(0.07)$ & -0.19 & 0.85 & $-0.16,0.14$ & -0.03 \\
\hline Baseline anxiety & $-0.01(0.06)$ & -0.20 & 0.85 & $-0.13,0.10$ & -0.04 \\
\hline Total intracranial volume ${ }^{a}$ & $1.78(1.08)$ & 1.65 & 0.11 & $-0.42,3.98$ & 0.28 \\
\hline Baseline depression & $-0.01(0.07)$ & -0.16 & 0.87 & $-0.16,0.14$ & -0.03 \\
\hline Baseline anxiety & $-0.02(0.06)$ & -0.30 & 0.76 & $-0.13,0.10$ & -0.06 \\
\hline Total intracranial volume ${ }^{a}$ & $1.56(1.10)$ & 1.43 & 0.16 & $-0.67,3.82$ & 0.25 \\
\hline Right putamen ${ }^{a}$ & $-2.67(1.01)$ & -2.68 & 0.01 & $-4.74,-0.64$ & -0.43 \\
\hline
\end{tabular}

Note: step I predicting 3-month anhedonia severity is the same for the left and right putamen; Mood and Feelings Questionnaire= depression; Multidimensional Anxiety Scale for Children = anxiety; Snaith-Hamilton Pleasure Scale= anhedonia.

ancluded standardized values as variables.

Table 3 Putamen Volume Moderates The Relationship Between Peer Feedback And Anhedonia Severity At The 3-Month Follow-Up Assessment $(n=37)$

\begin{tabular}{|c|c|c|c|c|}
\hline & $b(\mathrm{SE})$ & $t$ & p & $\mathrm{Cl}_{95}$ \\
\hline \multicolumn{5}{|c|}{ Left putamen model: $F(7,29)=7.66, p<0.001 ; R^{2}=0.59$} \\
\hline Baseline anhedonia & $0.40(0.14)$ & 2.87 & 0.01 & $0.11,0.68$ \\
\hline Baseline depression & $-0.04(0.07)$ & -0.50 & 0.62 & $-0.18,0.11$ \\
\hline Baseline anxiety & $-0.00 \mid(0.06)$ & -0.01 & 0.99 & $-0.12,0.12$ \\
\hline $\begin{array}{l}\text { Total intracranial } \\
\text { volume }^{\mathrm{a}}\end{array}$ & $1.74(0.92)$ & 1.89 & 0.07 & $-0.15,3.62$ \\
\hline Accept & $-0.06(0.06)$ & -0.95 & 0.35 & $-0.18,0.07$ \\
\hline Left putamen ${ }^{a}$ & $-2.97(0.84)$ & -3.55 & 0.001 & $-4.68,-1.26$ \\
\hline Accept $\times$ left putamen ${ }^{a}$ & $0.16(0.07)$ & 2.21 & 0.03 & $0.01,0.30$ \\
\hline \multicolumn{5}{|c|}{ Right putamen model: $F(7,29)=13.78, p<0.001 ; R^{2}=0.57$} \\
\hline Baseline anhedonia & $0.36(0.14)$ & 2.63 & 0.01 & $0.08,0.64$ \\
\hline Baseline depression & $-0.03(0.06)$ & -0.50 & 0.62 & $-0.16,0.10$ \\
\hline Baseline anxiety & $-0.01(0.05)$ & -0.11 & 0.91 & $-0.11,0.10$ \\
\hline $\begin{array}{l}\text { Total intracranial } \\
\text { volume }^{\mathrm{a}}\end{array}$ & $1.52(0.85)$ & 1.80 & 0.08 & $-0.21,3.26$ \\
\hline Accept & $-0.05(0.06)$ & -0.90 & 0.38 & $-0.18,0.07$ \\
\hline Right putamen ${ }^{\mathrm{a}}$ & $-2.82(0.65)$ & -4.32 & 0.0002 & $-4.15,-1.48$ \\
\hline Accept $\times$ right putamen ${ }^{a}$ & $0.14(0.06)$ & 2.27 & 0.03 & $0.01,0.27$ \\
\hline
\end{tabular}

Note: accept/reject $=$ subjective rating on the chatroom task after peer feedback (0-100); Mood and Feelings Questionnaire = Depression; Multidimensional Anxiety Scale for Children=Anxiety; Snaith-Hamilton Pleasure Scale $=$ anhedonia.

ancluded standardized values as variables.
( $p s>0.37)$. Further, no significant moderation effects emerged when testing whether caudate ( $p s>0.32$ ) or nucleus accumbens $(p>0.39)$ volume strengthened the association between peer acceptance and anhedonia. Similarly, no significant interactions emerged when testing caudate ( $p$ s $>0.52$ ) or nucleus accumbens $(p>0.65)$ volume and peer rejection.

\section{Secondary Analyses: Testing Specificity}

To test the specificity of our hypotheses, we conducted secondary analyses with depression and anxiety symptoms as outcome variables.

Cross-sectional analyses. When predicting baseline depression symptoms, anhedonia, anxiety, and total intracranial volume were included as covariates. No significant associations emerged with the putamen $(p s>0.51)$, caudate $(p s>0.66)$, and nucleus accumbens $(p s>0.32)$ volume. When testing associations with anxiety symptoms, anhedonia, depression, and total intracranial volume were included as covariates. No significant associations with putamen $(p s>0.70)$, caudate $(p s>0.63)$, and nucleus accumbens ( $p s>0.17)$ volume emerged.

Prospective analyses. In models predicting 3-month symptoms, baseline symptoms and total intracranial volume were included as covariates. No significant effects emerged when testing the relationship between putamen $(p s>0.68)$, caudate $(p s>0.41)$, and nucleus accumbens $(p s>0.27)$ volume and depressive symptoms. Similarly, no significant effects were 
observed between 3-month anxiety and putamen ( $p s>0.37)$, caudate $(p s>0.46)$, and nucleus accumbens $(p s>0.45)$ volume.

Moderation analyses. We also tested whether striatal volume moderated the relationship between peer feedback and depressive symptoms at the 3-month follow-up assessment. There were no significant moderation effects for peer acceptance $(p s>0.14)$ or peer rejection ( $p s>0.11)$. When testing whether striatal volume moderated the relationship between peer feedback and anxiety symptoms at the 3-month follow-up, no significant effects emerged for peer acceptance $(p s>0.10)$ or peer rejection $(p s>0.74)$.

\section{DISCUSSION}

Anhedonia is a transdiagnostic marker of risk for mental illness, and there is considerable interest in identifying neural mechanisms that contribute to the emergence of anhedonic symptoms. Three principal findings emerged. First, in crosssectional analyses, smaller right nucleus accumbens volume correlated with anhedonic symptoms. Second, reduced left and right putamen volume prospectively predicted 3-month anhedonia severity while controlling for baseline symptoms. Highlighting the specificity of these findings, no significant prospective relationships emerged among caudate or nucleus accumbens volume and subsequent anhedonia severity. Third, a blunted response to peer acceptance was associated with anhedonia severity, but only among youth with smaller putamen volume. Collectively, among the psychiatrically healthy adolescents, striatal volume specifically conferred vulnerability to anhedonia. As a result, these findings may inform early identification of individuals at high risk for mental illness.

Whereas prior research in adults has found an inverse correlation between caudate volume and anhedonia severity (Harvey et al, 2007; Pizzagalli et al, 2009), we found that reduced putamen volume-accounting for $11-14 \%$ of the variance-predicted anhedonia severity at the 3-month follow-up assessment. This raises the question as to whether there are developmental differences to consider when testing the relationship between brain volume abnormalities and anhedonia symptoms. The putamen is a structure of the forebrain that regulates motor planning (Haber, 2003; Monchi et $a l, 2006)$ and has been implicated in positive prediction errors (Pessiglione et al, 2006). Reduced putamen activation also is associated with anticipatory reward deficits (Kumar et al, 2014), particularly in the context of stress (Porcelli et al, 2012). One possibility is that the initial onset of anhedonia in youth is characterized by motor alterations, including reduced energy and diminished motivation. As anhedonia intensifies, increased behavioral withdrawal may lead to the emergence of broader anhedonia (eg, social anhedonia) and other psychiatric symptoms. In general, the transition through puberty is marked by a normative decrease in putamen volume (Goddings et al, 2014), which may account for why the putamen-anhedonia association is present in adolescents but not in adults. This possibility, however, warrants further research. Alternatively, anhedonia is not a monolithic entity-encompassing anticipatory, consummatory, and motivational processes-but most current self-report scales of anhedonia fail to consider such heterogeneity. Given this inherent heterogeneity, it is reasonable to suspect that putamen and caudate volume may be associated with different facets of anhedonia. Reconciling these issues may yield key insights into anhedonia as a transdiagnostic marker for wide-ranging psychiatric illnesses.

Prior functional MRI research using peer feedback paradigms has implicated the putamen (Guyer et al, 2009, 2012), however, this is the first study to test whether striatal volume moderates the relationship between peer feedback and subsequent anhedonia symptoms. Results indicated that a blunted response to peer acceptance predicted higher levels of anhedonia, but only among adolescents with smaller putamen volume. Thus, reduced putamen volume may reflect a trait-like characteristic that increases affective reactivity to social feedback; particularly as subcortical volumetric abnormalities impair hedonic encoding and reduce motivation to engage in pleasurable experiences (Harvey et al, 2007). A blunted response to peer acceptance did not predict anhedonia (see Table 3), but rather the interaction of this social feedback response and reduced putamen volume led to anhedonia. Seemingly, youth who do not experience pleasure in otherwise positive social interactions, may be more inclined to socially isolate, which in turn, may increase risk for anhedonia and associated psychiatric illness onset.

\section{Limitations}

Our findings should be interpreted in the context of several limitations. First, the study included females, and it is unclear whether results generalize to male adolescents. Second, anhedonia severity was in the non-clinical range. A key unanswered question is whether anhedonia leads to disorder onset and relatedly, if these symptoms impact adolescent quality of life. Third, striatal structure experiences change during adolescence (Goddings et al, 2014); accordingly, research should examine how these dynamic alterationsthrough the use of repeated MRI structural assessments over time-relate to symptom onset. Last, it is unclear why crosssectional (nucleus accumbens) and prospective (putamen) analyses linked different striatal regions to anhedonia, especially since these symptoms were assessed with the same instrument. Future studies, including those probing different facets of anhedonia, will be needed to resolve these open questions.

\section{Summary}

Anhedonia is implicated in the onset of mental illness (Pizzagalli, 2014), treatment non-response (McMakin et al, 2012), and suicidal behaviors (Auerbach et al, 2015). Identifying neuroanatomical markers that confer risk for anhedonia may lead to the development of preventativeintervention programs aimed at helping youth develop compensatory strategies to combat the emergence of these debilitating symptoms.

\section{FUNDING AND DISCLOSURE}

Over the past 3 years, Dr Pizzagalli has received consulting fees from Akili Interactive Labs, BlackThorn Therapeutics, 
Pfizer and Posit Science, for activities unrelated to the current research. The remaining authors declare no conflict of interest.

\section{ACKNOWLEDGMENTS}

The project was supported through funding from the National Institute of Mental Health (NIMH) K23MH097786 (RPA), NIMH R01MH101521 (DAP), NIMH R01MH068376 (DAP), The Dana Foundation: Clinical Neuroscience Research Grant (RPA, DAP), Klingenstein Third Generation Foundation Adolescent Depression Fellowship (RPA), and Tommy Fuss Fund (RPA, DAP). DAP was further supported by NIMH R37MH068376. The content is solely the responsibility of the authors and does not necessarily represent the official views of the National Institutes of Health or NIMH.

\section{REFERENCES}

Angold A, Costello EJ, Messer SC, Pickles A (1995). Development of a short questionnaire for use in epidemiological studies of depression in children and adolescents. Int J Methods Psychiatr Res 5: 237-249.

Auerbach RP, Admon R, Pizzagalli DA (2014). Adolescent depression: stress and reward dysfunction. Harv Rev Psychiatry 22: 139-148.

Auerbach RP, Ho M-H (2012). A cognitive-interpersonal modle of adolescent depression: the impact of family conflict and depressogenic cognitive styles. J Clin Child Adolesc Psychol 41: 792-802.

Auerbach RP, Millner AJ, Stewart JG, Esposito EC (2015). Identifying differences between depressed adolescent suicide ideators and attempters. J Affect Disord 186: 127-133.

Avenevoli S, Swendsen J, He JP, Burstein M, Merikangas KR (2015). Major depression in the national comorbidity survey-adolescent supplement: prevalence, correlates, and treatment. J Am Acad Child Adolesc Psychiatry 54: 37-44 e32.

Delgado MR (2007). Reward-related responses in the human striatum. Ann N Y Acad Sci 1104: 70-88.

Dillon DG, Holmes AJ, Birk JL, Brooks N, Lyons-Ruth K, Pizzagalli DA (2009). Childhood adversity is associated with left basal ganglia dysfunction during reward anticipation in adulthood. Biol Psychiatry 66: 206-213.

Fawcett J, Scheftner WA, Fogg L, Clark DC, Young MA, Hedeker D et al (1990). Time-related predictors of suicide in major affective disorder. Am J Psychiatry 147: 1189-1194.

Fischl B, van der Kouwe A, Destrieux C, Halgren E, Ségonne F, Salat DH et al (2004). Automatically parcellating the human cerebral cortex. Cereb Cortex 14: 11-22.

Forbes EE, Dahl RE (2005). Neural systems of positive affect: relevance to understanding child and adolescent depression? Dev Psychopathol 17: 827-850.

Goddings AL, Mills KL, Clasen LS, Giedd JN, Viner RM, Blakemore SJ (2014). The influence of puberty on subcortical brain development. Neuroimage 88: 242-251.

Guyer AE, Choate VR, Pine DS, Nelson EE (2012). Neural circuitry underlying affective response to peer feedback in adolescence. Soc Cogn Affect Neurosci 7: 81-92.

Guyer AE, McClure-Tone EB, Shiffrin ND, Pine DS, Nelson EE (2009). Probing the neural correlates of anticipated peer evaluation in adolescence. Child Dev 80: 1000-1015.

Haber SN (2003). The primate basal ganglia: parallel and integrative networks. J Chem Neuroanat 26: 317-330.

Haber SN, Knutson B (2010). The reward circuit: linking primate anatomy and human imaging. Neuropsychopharmacology 35: 4-26.
Harvey PO, Pruessner J, Czechowska Y, Lepage M (2007). Individual differences in trait anhedonia: a structural and functional magnetic resonance imaging study in non-clinical subjects. Mol Psychiatry 12703 767-775.

Hayes AF (2013). Introduction to mediation, moderation, and conditional process analysis: a regression-based approach. The Guilford Press: New York, NY, USA xvii: 507.

Kaufman J, Birmaher B, Brent D, Rao U, Flynn C, Moreci P et al (1997). Schedule for affective disorders and schizophrenia for school-age children-present and lifetime version (K-SADS-PL): initial reliability and validity data. J Am Acad Child Adolesc Psychiatry 36: 980-988.

Katz RJ, Roth KA, Carroll BJ (1981). Acute and chronic stress effects on open field activity in the rat: implications for a model of depression. Neurosci Biobehav Rev 5: 247-251.

Kempton MJ, Salvador Z, Munafo MR, Geddes JR, Simmons A, Frangou $S$ et al (2011). Structural neuroimaging studies in major depressive disorder. Meta-analysis and comparison with bipolar disorder. Arch Gen Psychiatry 68: 675-690.

Kessler RC (2012). The costs of depression. Psychiatr Clin North Am 35: 1-14.

Koolschijn PC, van Haren NE, Lensvelt-Mulders GJ, Hulshoff Pol HE, Kahn RS (2009). Brain volume abnormalities in major depressive disorder: a meta-analysis of magnetic resonance imaging studies. Hum Brain Mapp 30: 3719-3735.

Kumar P, Berghorst LH, Nickerson LD, Dutra SJ, Goer FK, Greve DN et al (2014). Differential effects of acute stress on anticipatory and consummatory phases of reward processing. Neuroscience 266: $1-12$.

Lucas LR, Celen Z, Tamashiro KL, Blanchard RJ, Blanchard DC, Markham $C$ et al (2004). Repeated exposure to social stress has long-term effects on indirect markers of dopaminergic activity in brain regions associated with motivated behavior. Neuroscience 124: 449-457.

March JS, Parker JD, Sullivan K, Stallings P, Conners CK (1997). The Multidimensional Anxiety Scale for Children (MASC): factor structure, reliability, and validity. I Am Acad Child Adolesc Psychiatry 36: 554-565.

McCabe C, Cowen PJ, Harmer CJ (2009). Neural representation of reward in recovered depressed patients. Psychopharmacology (Berl) 205: 667-677.

McMakin DL, Olino TM, Porta G, Dietz LJ, Emslie G, Clarke G et al (2012). Anhedonia predicts poorer recovery among youth with selective serotonin reuptake inhibitor treatment-resistant depression. J Am Acad Child Adolesc Psychiatry 51: 404-411.

Mehta MA, Gore-Langton E, Golembo N, Colvert E, Williams SC, Sonuga-Barke E (2010). Hyporesponsive reward anticipation in the basal ganglia following severe institutional deprivation early in life. J Cogn Neurosci 22: 2316-2325.

Monchi O, Petrides M, Strafella AP, Worsley KJ, Doyon J (2006). Functional role of the basal ganglia in the planning and execution of actions. Ann Neurol 59: 257-264.

Nock MK, Kazdin AE (2002). Examination of affective, cognitive, and behavioral factors and suicide-related outcomes in children and young adolescents. J Clin Child Adolesc Psychol 31: $48-58$.

O'Doherty J, Dayan P, Schultz J, Deichmann R, Friston K, Dolan RJ (2004). Dissociable roles of ventral and dorsal striatum in instrumental conditioning. Science 304: 452-454.

O'Doherty JP (2004). Reward representations and reward-related learning in the human brain: insights from neuroimaging. Curr Opin Neurobiol 14: 769-776.

O'Doherty JP, Dayan P, Friston K, Critchley H, Dolan RJ (2003). Temporal difference models and reward-related learning in the human brain. Neuron 38: 329-337.

Pagnoni G, Zink CF, Montague PR, Berns GS (2002). Activity in human ventral striatum locked to errors of reward prediction. Nat Neurosci 5: 97-98. 
Pessiglione M, Seymour B, Flandin G, Dolan RJ, Frith CD (2006). Dopamine-dependent prediction errors underpin reward-seeking behaviour in humans. Nature 442: 1042-1045.

Pizzagalli DA (2014). Depression, stress, and anhedonia: toward a synthesis and integrated model. Annu Rev Clin Psychol 10: 393-423.

Pizzagalli DA, Holmes AJ, Dillon DG, Goetz EL, Birk JL, Bogdan R et al (2009). Reduced caudate and nucleus accumbens response to rewards in unmedicated individuals with major depressive disorder. Am J Psychiatry 166: 702-710.

Porcelli AJ, Lewis AH, Delgado MR (2012). Acute stress influences neural circuits of reward processing. Front Neurosci 6: 157.

Rudolph KD (2008). Developmental influences on interpersonal stress generation in depressed youth. J Abnorm Psychol 117: 673-679.

Schultz W (2016). Dopamine reward prediction-error signalling: a two-component response. Nat Rev Neurosci 17: 183-195.

Schultz W, Dayan P, Montague PR (1997). A neural substrate of prediction and reward. Science 275: 1593-1599.
Snaith RP, Hamilton M, Morley S, Humayan A, Hargreaves D, Trigwell P (1995). A scale for the assessment of hedonic tone the Snaith-Hamilton Pleasure Scale. Br J Psychiatry 167: 99-103.

Spijker J, Bijl RV, de Graaf R, Nolen WA (2001). Determinants of poor 1-year outcome of DSM-III-R major depression in the general population: results of the Netherlands Mental Health Survey and Incidence Study (NEMESIS). Acta Psychiatr Scand 103: $122-130$

Tanner JM, Davies PSW (1985). Clinical longitudinal standards for height and height velocity for North American children. J Pediatr 107: 317-329.

Treadway MT, Zald DH (2011). Reconsidering anhedonia in depression: lessons from translational neuroscience. Neurosci Biobehav Rev 35: 537-555.

Yorbik O, Birmaher B, Axelson D, Williamson DE, Ryan ND (2004). Clinical characteristics of depressive symptoms in children and adolescents with major depressive disorder. J Clin Psychiatry 65: 1654-1659, quiz 1760-1651. 